\title{
A Study On OFDM In Mobile Ad Hoc Network
}

\author{
Malik Nasereldin Ahmed \\ Computer Science \& Information system \\ University Technology Malaysia \\ Johor Bahru - Malaysia
}

\author{
Abdul Hanan Abdullah \\ Computer Science \& Information system \\ University Technology Malaysia \\ Johor Bahru - Malaysia
}

\author{
Satria Mandala \\ Computer Science \& Information system \\ The state of Islamic University of \\ MALIKI-Indonesia
}

\begin{abstract}
Orthogonal Frequency Division Multiplexing (OFDM) is the physical layer in emerging wireless local area networks that are also being targeted for ad hoc networking. OFDM can be also exploited in ad hoc networks to improve the energy performance of mobile devices. It is important in wireless networks because it can be used adaptively in a dynamically changing channel. This study gives a detailed view about OFDM and how it is useful to increase the bandwidth. This paper also gives an idea about how OFDM can be a promising technology for high capacity wireless communication.
\end{abstract}

Keywords- Ad hoc; OFDM; MANET.

\section{INTRODUCTION}

\section{A. Ad hoc Networks}

Ad hoc networks are a new paradigm of wireless communication for mobile hosts where node mobility causes frequent changes in topology. Mobile nodes that are within each other's radio range communicate directly via wireless links called peer to peer or single hop ad hoc network. If destination mobile node is out of range of the source mobile node, other nodes in the range of the source and destination acts as router to transmit packets between source and destination and this is called multi - hop ad hoc networks. The figures 1 and 2 show single and multi-hop ad hoc network. Ad hoc networks are self-configurable and autonomous systems consisting of routers and hosts, which are able to support mobility and organize themselves arbitrarily. Moreover, the ad hoc network can be either constructed or destructed quickly and autonomously without any administrative server or infrastructure. If the nodes of ad hoc networks are mobile and with wireless communication to maintain the connectivity, it is known as mobile ad hoc network (MANET) and require an extremely flexible technology for establishing communications in situations which demand a fully decentralized network without any fixed base stations, such as battle fields, military applications and other emergency and disaster situations.

There are some challenges in mobile environments like limitations of the wireless network, packet loss due to transmission errors, variable capacity links, frequent disconnections / partitions, limited communication bandwidth and broadcast nature of the communications. In addition, limitation imposed by mobility dynamically changing topologies / routers lacks of mobility awareness by system / applications. Limitations of the mobile computer such as short battery lifetime and limited capacities will create more problems for the transmission.

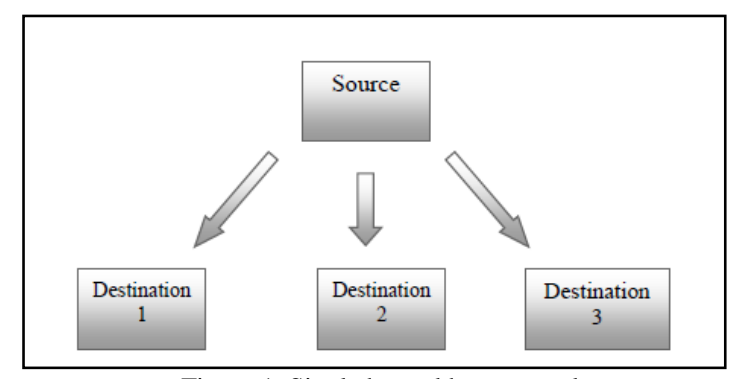

Figure 1. Single hop ad hoc network

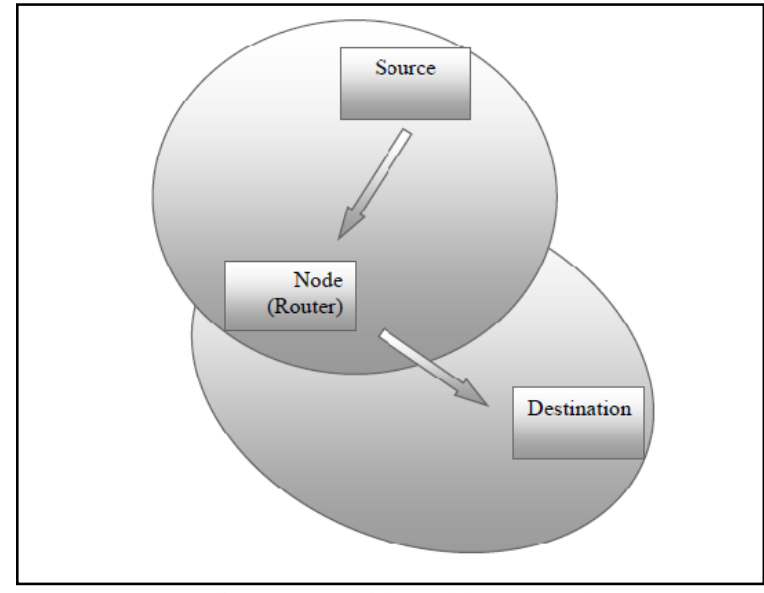

Figure 2. Multi hop ad hoc network

Ad hoc network has some problems include routing, broadcasting, multicasting, geo casting, clustering, area monitoring, data and service access. Topology control problems include neighbor discovery, topology construction and maintenance, activity scheduling, position discovery, partition detection, location updates, and network management. In addition, there are two fundamental problems network information capacity and minimum energy per bit.

Multiplexing or Muxing, is the process where multiple analog or digital signals combined in one signal over shared medium. For example, it is possible to transfer many phone calls in one signal at the same time. At the receiver side, the combined signals must be separate into the original signal. This process is called Demultiplexing.

Multiplexing techniques may be divided into many different types and they are space division multiplexing 
(SDM), frequency division multiplexing (FDM), time division multiplexing (TDM) and code division multiplexing (CDM).

\section{A. OFDM}

OFDM is a modulation technique as well as multiplexing technique as it is divide a single high data rate stream into a number of lower rate streams that are data transmitted simultaneous over some narrow sub channel. OFDM technique offering better use of ad hoc network like intersymbol interference (ISI), OFDM avoids this problem by sending many low speed transmissions simultaneously and increase the network throughput.[1][2][3]

In OFDM, the sub carrier frequencies are chosen so that the sub carriers are orthogonal to each other. This greatly simplifies the design of both the transmitter and the receiver. Unlike conventional Frequency Division Multiplexing (FDM), a separate filter for each sub channel is not required. The orthogonality allows high spectral efficiency with a total symbol rate near the Nyquist rate for the equivalent baseband signal. Almost the whole available frequency band can be utilized. [4]

In conventional OFDM systems most of the approaches to combat ICI are towards using frequency synchronization and interference cancellation. They are usually very complex and sometimes there is loss in bandwidth efficiency OFDM system create high data rate with long symbol duration by combining low data rate. It reduces equalization complexity by implementing with Inverse Fast Fourier Transform (IFFT) at the transmitter and Fast Fourier Transform (FFT) at the receiver that converts the wide band signal affected by frequency selective fading into $\mathrm{N}$ narrowband flat fading signals.[8][9] The beneficial since OFDM enables support of more antennas and large band widths since it simplifies equalization dramatically in MIMO system. Hence, the available bandwidth is utilized very efficiently in OFDM systems. The figure 3 shows the physical model of the design $[10][11][12]$.

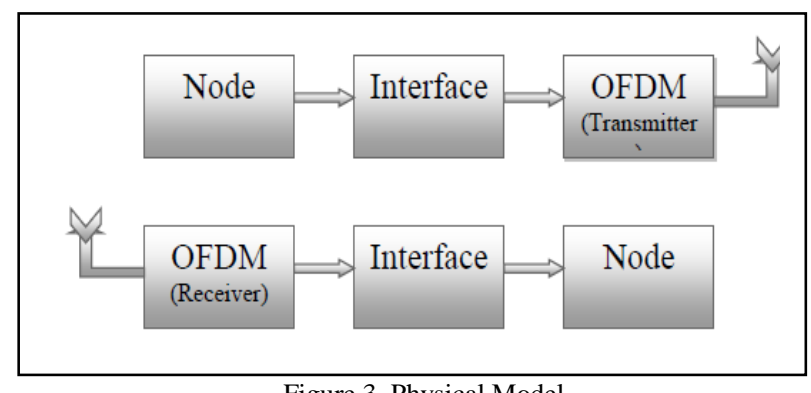

Figure 3. Physical Model

\section{REVIEW OF THE LITERATURE}

Dandan wang and Uf tureli[7] proposed a new transceiver architecture with MIMO - OFDM in the physical layer and an adaptive multiple antennas receiver initiated busy tone medium access to improve the performance of ad hoc networks. They have presented both theoretical and numerical analysis of the throughput and delay.

Gavin yeng et.al.[6] presented a detailed OFDM and channel modeling on the performance evaluation of higher layer protocols. The result of the model given by the author, show that device and wireless channel can impact packet delivery ratios and even point out a deficiency of the auto rate fall back protocol.

Swati chowduri et.al.[5] proposed a model which implements a MIMO- OFDM for MIMO based mobile ad hoc network and compared its bit error rate with different modulation technique. They have described different approaches for channel estimation and data detection. They have evaluated the performance of OFDM system using phase shift keying and quadrature amplitude modulation with OFDM using generalized gamma fading distribution.

Kamol kaemarungsi and prashant Krishnamurthy[4] studies shows that adaptive OFDM can be also exploited in ad hoc networks to improve the energy performance of mobile devices. They have evaluated the improvement in performance of adaptive OFDM over non - adaptive OFDM in ad hoc networks using simulations. Their study shows that how much of energy can be preserved by employing adaptive OFDM on the physical layer and how the channel information gain from the loading algorithm will help improve the energy preservation of ad hoc wireless network.

In this paper we have done a simulation to show that the OFDM got the characteristics to improve the bandwidth in the MANET. Moreover the study reveals the advantages of OFDM over other multiplexing technique.

\section{SimUlATION AND RESUltS}

OFDM is a physical layer encoding technology for transmitting signals through the RF. This method breaks one high - speed data carrier into several lower speed carriers. These are in turn transmitted in parallel across that particular RF spectrum. The 802.11a subcommittee has elected to use the transmission technique for their standard in the $5 \mathrm{GHz}$ unlicensed national information infrastructure bands. OFDM also used in the $2.4 \mathrm{GHz}$ ISM band as the physical layer standard for the $802.11 \mathrm{~g}$ standard. The simulation design contains three parts. The figure 3 shows the detailed view of the design.

Node: It is the receiving end; it may be a laptop, desktop or a mobile. In the MATLAB 'Signal generator' selected to act as a node and it generates continuous square pulse at 1 amplitude and $1 \mathrm{~Hz}$ frequency.

Interface: Discrete input signal at 0.5 intervals.

OFDM: This block contains three units. P / S to convert signals from parallel to serial; Inverse Fast Fourier Transform (IFFT) is useful for OFDM because it generates samples of a waveform with frequency components satisfying orthogonally conditions. The following Fourier transform shows the inphase and quadrature components.

$$
s(t)=\sum_{n=0}^{N-1}\left[a(n) \cos \left(\omega_{n} t\right)+b(n) \sin \left(\omega_{n} t\right)\right](1)
$$

$a(n)-$ is a real sequence representing the in-phase component. 
$b(n)$ - is a real sequence representing the quadrature component.

Fourier Fast Transform ( FFT) : A FFT is an efficient algorithm to compute the Discrete Fourier Transform ( DFT ) and its inverse. There are many distinct FFT algorithms involving a wide range of mathematics from simple complex number arithmetic to group theory and number theory.

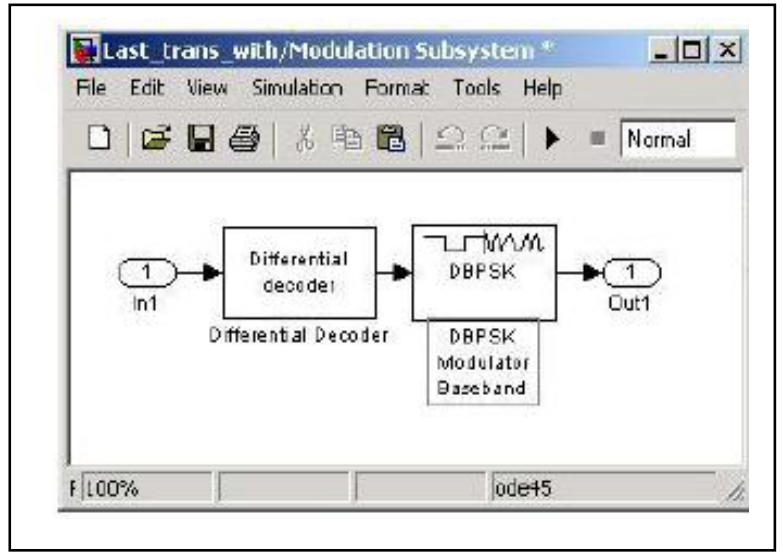

Figure 4. Modulation and Decoder Subsystem

\section{A. System Transmitter}

Transmission system generates digital signals and it will be quantized and enter into the OFDM system. After that the signal sends through radio wave. In the simulation, we have proved that OFDM increases bandwidth. Bandwidth describes the signal media carrier and the extent of use. This can be known by discovering the signal in the frequency domain and measuring the distance between the $\mathrm{f} \quad 1$ and $\mathrm{f} 2$ at $-3 \mathrm{~dB}$. The following figure5 shows the OFDM transmitter and figure 6 shows the normal increased bandwidth.

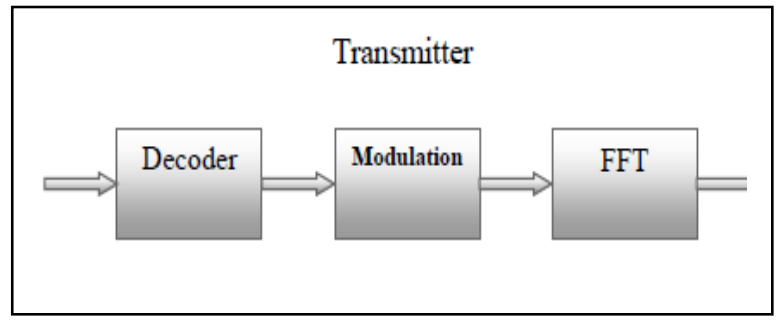

Figure. 5 OFDM Transmitter

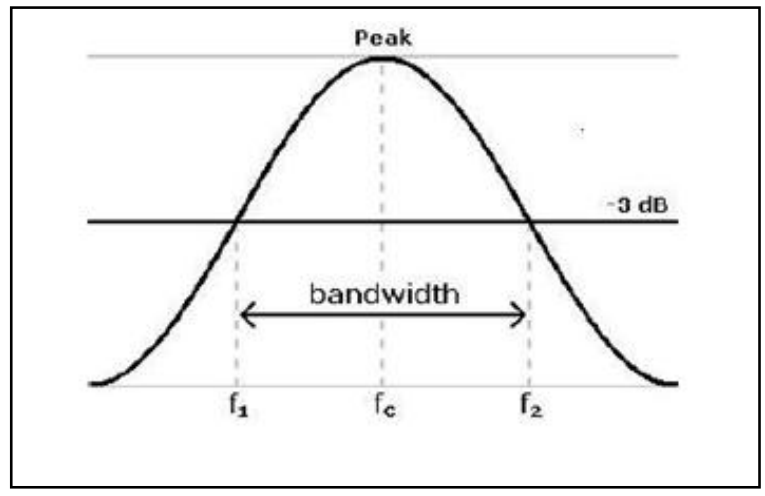

Figure 6. Normal Bandwidth
We have made this simulation to prove that the OFDM increases the bandwidth. First part of our simulation is transmitter without OFDM. The following figures show the transmitter without OFDM and output of the system.

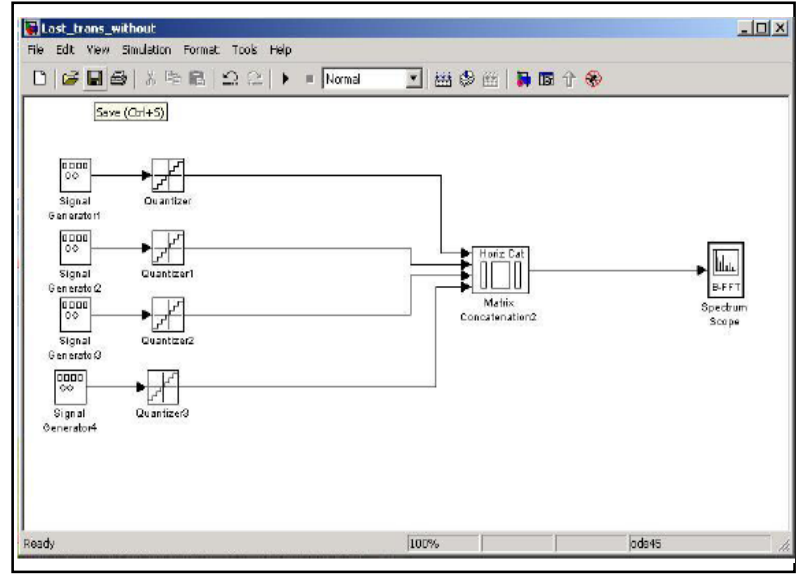

Figure 7. Transmitter without OFDM

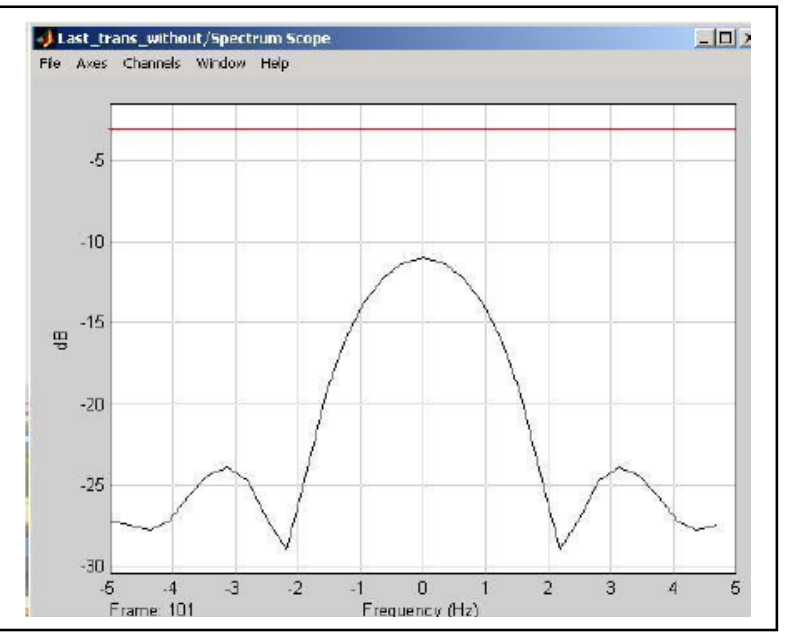

Figure 8. Output of the Transmitter without OFDM

The straight red line in the figure represents the $-3 \mathrm{~dB}$. It is clear that bandwidth did not reach the maximum level. The same process with OFDM is shown below.

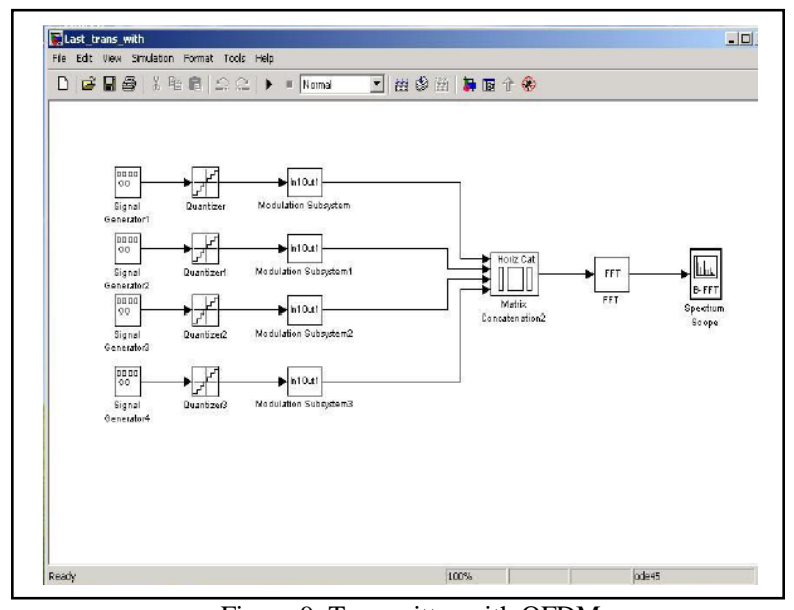

Figure 9. Transmitter with OFDM 


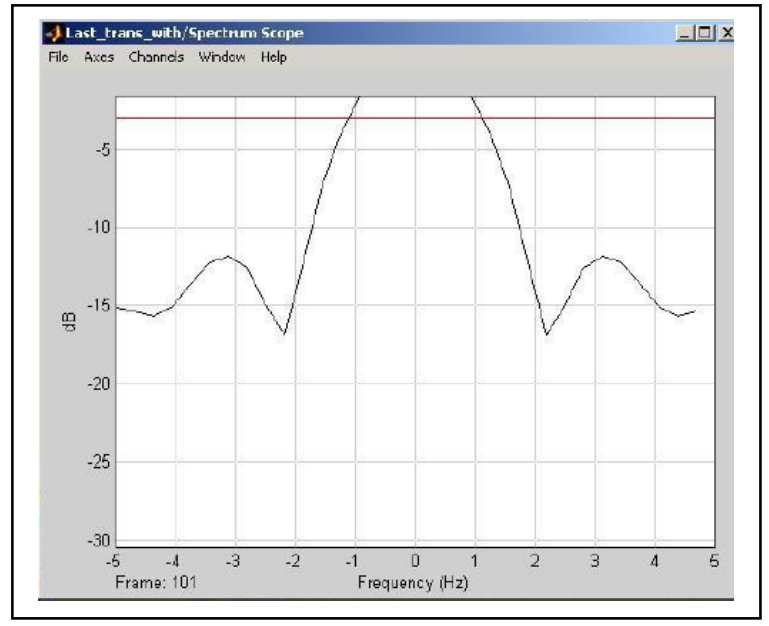

Figure 10. Output of the Transmitter with OFDM

The output shows clearly that the OFDM increases the bandwidth.

\section{CONCLUSION}

This paper has presented on the effects of detailed OFDM and its advantages over other multiplexing techniques. The results show that the OFDM acts as an accelerator to increase the bandwidth.

Adaptive OFDM system has optimum throughput performance for low and high mobility conditions. OFDM is a promising technology suitable for high capacity wireless communications and it is computationally efficient by using FFT techniques to implement the modulation and demodulation functions.

\section{REFERENCE}

[1] Shan Chu, Xin Wang, “ Opportunistic and Co operative spatial multiplexing in MIMO Ad-hoc networks:, IEEE / ACM Trans. On Networking. Vol.18, no.5, Oct 2010.
[2] B.Sathish kumar, K.R.Shankar Kumar, R.Radhakrishnan, “ An efficient inter carrier interference cancellation schemes for OFDM systems", IJCSIS Vol.6, No.3, 2009.

[3] D.K.Sharma, A.Mishra and Rajiv Savena, "Analog \& Digital Modulation Technique: An overview", International Journal of Computer Science and Communication technologies, Vol 3, No.1, July 2010.

[4] Kamol Kaemarungsi, Prashant Krishnamurthy," On the use of Adaptive OFDM to preserve energy in Ad-hoc wireless networks", Proceedings of 13th MPRG, Page(s): 1 -4.

[5] Swati Chowdhuri, Dr.Arun kumar Mondal, Dr.P.K.Baneerjee," Implementation of MIMO - OFDM system in mobile Ad-Hoc networks", International journal of Engineering Research and Applicaition:, Vol.1, Iss.3, Page(s): 753 - 757.

[6] Gavin Yeung, Mineo Takai, Rajive Bagrodia, Alireza Mehrnia, Babak Daneshrad,"Detailed OFDM modeling in network simulation of mobile Ad-Hoc networks.

[7] Dandan Wang, UF Tureli, “ Joint MIMO - OFDM and MAC design for broadband multi hop Ad Hoc networks", DRAFT, Nov. 1, 2005.

[8] P.H.Moose, " A technique for orthogonal frequency division multiplexing frequency offset correction", IEEE transactions on communications Vol 42, No.10, 1994.

[9] H.Yang, " A Road to future broadband wireless access: MIMO - OFDM based Air Interface", Communications Magazine, Vol.43, No.1, Jan 2005, Page(s): $53-60$.

[10] R.Rom and M.Sidi, “ Multiple Access Protocols Performance and Analysis", Springer Verilog 1990.

[11] IEEE standard for wireless LAN Medium Access Control (MAC) and physical layer specifications, Nov. 1997, P802.11.

[12] T.Tang, M.Park, R.W.Heath and S.M. Nettles, “ A joint MIMO OFDM transceiver and MAC design for mobile Ad hoc networking, International workshop on wireless ad - hoc networks 2004.

[13] Q.Zhao and L.Tang, "A multiqueue service room MAC protocol for wireless networks with multipacket reception", IEEE / ACM trans. Networking Vol.11, No.1, Feb 2003 Page(s):125 - 137.

[14] Charles.E.Perkins, Elizabeth.M.Royer, "Ad-hoc on demand distance vector routing", in proceedings of 2nd IEEE workshop on mobile computing systems and application. Page(s): 90 - 100, 1999.

[15] Gavin Holland, Nitin Vaidya, Paramvir Bahl, “ A Rate Adaptive MAC protocol for multi hop wireless networks", in proceedings of mobicom 2001, July 2001.

[16] Mineo Takai, Jay Martin, Rajive Bagrodia, "Effects of wireless physical layer modeling in mobile Ad-hoc network", in proceedings of mobihoc 2001, page(s): 87 - 94, Oct 2001. 